\title{
Transfer of contextual control across similarly trained conditioned stimuli
}

\author{
DALE SWARTZENTRUBER \\ University of Pennsylvania, Philadelphia, Pennsylvania
}

\begin{abstract}
Two autoshaping experiments with pigeons examined contextual control of responding to conditioned stimuli (CSs) when separate phases of reinforcement and nonreinforcement occurred in different contexts. In Experiment 1, a CS was first conditioned in Context A prior to nonreinforcement in Context B. In Experiment 2, a conditional discrimination reversal procedure was employed in which one CS was first reinforced in A prior to nonreinforcement in B, and another CS was first nonreinforced in A and then reinforced in B. In both experiments, the extent of contextual control was assessed by testing the CSs in A and B. The CS specificity of control was assessed by examining control of CSs that had been treated like the original CSs, but in a different pair of contexts. The results show that contexts control responding to CSs through a CS-specific mechanism, as well as through a mechanism that is independent of the identity of the CS. However, the extent to which control is mediated by a CS-independent mechanism depends on the training history of the CS.
\end{abstract}

In many Pavlovian and operant learning paradigms, responding to a stimulus can depend importantly on the contextual or background cues that are present. An issue that has received considerable recent attention is the mechanism that is involved in this contextual control of performance.

The potential mechanisms that have been proposed can be divided into two main classes. The classes differ in whether the effect of context is attributed to a general acquired property of the context that is independent of a given conditioned stimulus (CS), or to a specific feature of the context/CS combination that has been experienced during training.

The first view asserts that responding to a CS in a particular context is determined by the individual properties of the CS and context. For example, this view encompasses the popular account that learning about contexts and learning about localized CSs follow the same basic rules. During training, the contexts acquire simple excitatory and inhibitory associations with the unconditioned stimulus (US) that summate with those of the CS (see, e.g., Rescorla \& Wagner, 1972). In an alternative account within this same class of mechanism, the context and CS play different roles. The context may acquire the ability to modulate responding to CSs in a manner that is independent of the context's simple US associations (see, e.g.,

This research was supported by National Science Foundation Grant BNS 88-03514 to R. A. Rescorla and by National Institute of Mental Health postdoctoral training Grant MH 09896 to the author. I thank R. A. Rescorla and an anonymous reviewer for comments made on a draft of this manuscript, and S. E. Coldwell for computer simulations of Pearce's (1987) model. Address correspondence to the author at the Department of Psychology, Ohio Wesleyan University, Delaware, $\mathrm{OH}$ 43015 .
Rescorla, Durlach, \& Grau, 1985). This type of role for contexts is similar to a higher order modulatory role for discrete stimuli that has been advocated by both Rescorla (e.g., 1985) and Holland (e.g., 1989). The prediction common to both simple associative and modulatory views is that contextual control is not specific to the CS involved in training.

In another broad class of mechanisms, contextual control of CS performance depends on prior experience with that particular context/CS combination. For example, one proposal is that each combination is treated as a unique configural stimulus whose excitatory or inhibitory strength is reflected in performance. This view is well exemplified by a model of stimulus generalization recently proposed by Pearce (1987). Alternatively, contexts may control responding hierarchically by "setting the occasion" for a specific CS-US association. According to this conception, the context may affect responding by signaling the CS reinforcement contingency previously experienced in that context (see, e.g., Bouton, 1991; Bouton \& Swartzentruber, 1986; Holland, 1983). Within either view, the context's influence on responding to a CS is due to a prior treatment with that CS in that context.

Since these views differ in the extent to which contextual control is CS specific, one method for assessing the contribution of the various mechanisms is to examine responding to novel context/CS compounds. This strategy was used in the present experiments, in which the nature of contextual control was assessed with two different Pavlovian procedures. In Experiment 1, control was examined after simple Pavlovian excitatory conditioning and extinction occurred in different contexts. In Experiment 2, one CS was conditioned in one context and then extinguished in a different context, and another CS was first nonreinforced prior to conditioning in a different context. 
In each procedure, two issues were addressed. First, the extent to which the contexts controlled responding to the original CSs was examined by testing the CSs in the two training contexts. Responding in a manner appropriate to the reinforcement contingencies experienced in those contexts would suggest that the contexts exerted control over CS performance. The second issue concerned the CSspecific nature of this control. This was addressed by examining the contexts' ability to affect responding to CSs that had been treated similarly to the original CSs, but in a different pair of contexts. If contextual control is CS specific, one would expect to find little control over responding to these transfer CSs. However, if control results from a mechanism that is independent of CS identity, the contexts should influence responding to these CSs as well.

The mechanisms of contextual control in Pavlovian conditioning have been assessed by using a number of different preparations, including conditioned suppression with rats (see, e.g., Bouton \& King, 1983, 1986; Bouton \& Swartzentruber, 1986; Grahame, Hallam, Geier, \& Miller, 1990; Hall \& Honey, 1989, 1990; Lovibond, Preston, \& Mackintosh, 1984; Swartzentruber \& Bouton, 1988), appetitive conditioning with rats (see, e.g., Bouton \& Peck, 1989; Hall \& Honey, 1989; Swartzentruber, 1991; Wilson \& Pearce, 1989, 1990), and autoshaping with pigeons (see, e.g., Grau \& Rescorla, 1984; Honey et al., 1990; Rescorla et al., 1985). Transfer experiments from each preparation have yielded evidence for the contribution of CS-specific contextual processes; however, because the experiments were designed for a different purpose, many did not provide a sensitive assessment of the contribution of a CS-independent process.

In situations where contextual control has failed to transfer across CSs, transfer stimuli have typically been trained in a manner different from that of the original CS (see, e.g., Bouton \& King, 1986; Bouton \& Swartzentruber, 1986, 1989; Grahame et al., 1990). A more sensitive assessment of CS specificity is provided by matching the original and transfer CSs in all aspects except the identity of the contexts in which training occurs. In this manner, any difference in the extent to which the context controls responding to the original and transfer CSs during the test could only be due to the relation of the stimuli to the test context. The few experiments that have assessed transfer of contextual control across similarly trained CSs have provided evidence of transfer (e.g., Swartzentruber \& Bouton, 1988; Wilson \& Pearce, 1989, 1990). In the training procedure in those experiments, a CS was alternately reinforced and nonreinforced in different contexts. Transfer of control by either the reinforcement or the nonreinforcement context was found with a CS that had been trained similarly to the original CS, but in a different context.

This strategy was used in the present experiments to examine the CS specificity of control in an autoshaping preparation with pigeons. These experiments differed from previous ones in several respects. Instead of train- ing with alternating sessions of reinforcement and nonreinforcement, the procedure involved a single reversal of the reinforcement contingencies. As a result, it was possible to investigate the extent and nature of contextual control of CSs that are first reinforced and then nonreinforced, as well as CSs that are first nonreinforced and then reinforced. Furthermore, unlike previous designs, which focus on control by either the reinforcement or the nonreinforcement contexts alone, the present experiments assessed the extent and nature of control by both the conditioning and the extinction contexts concurrently.

\section{EXPERIMENT 1}

This experiment examined contextual control of responding to a CS that was first conditioned in one context and then extinguished in another (see Table 1). One CS (X) first received excitatory conditioning in Context A and then extinction in Context $\mathrm{B}$, whereas another $\mathrm{CS}(\mathrm{Z})$ received identical conditioning and extinction treatments in Contexts $\mathrm{C}$ and $\mathrm{D}$. Contextual control was assessed by examining differential responding to $\mathrm{X}$ and $\mathrm{Z}$ in their respective training contexts. The specificity of that control was determined by examining the ability of the contexts to control responding to the alternate CS. If contextual control is based on a mechanism that is independent of the specific context/CS combination used during training, the contexts should transfer control to the alternate $\mathrm{CS}$ in a manner similar to that shown to the original CS. However, if contextual control involves a process unique to the context/CS combinations used in original training, the contexts should exert little control over responding to the transfer CS.

\section{Method}

Subjects. The subjects were 16 female Carneaux pigeons, about 1 year old. They had participated in another autoshaping experiment with stimuli different from those used here. The subjects were housed in pairs and maintained at $80 \%$ of their free-feeding weights.

Apparatus. Eight identical operant chambers, each measuring $27 \times 27 \times 35 \mathrm{~cm}$, were used. One wall of each chamber was constructed of aluminum, and contained a $5 \times 5 \mathrm{~cm}$ food magazine centered $5 \mathrm{~cm}$ above the wire mesh floor. A $11.8 \times 14.5 \mathrm{~cm}$ response key, constructed of clear Lucite acrylic, was centered $9 \mathrm{~cm}$

Table 1

Design of Experiments 1 and 2

\begin{tabular}{|c|c|c|}
\hline \multicolumn{3}{|c|}{ Experiment 1} \\
\hline Conditioning & Extinction & Test in $A, B, C$, and $D$ \\
\hline $\begin{array}{l}\text { A: } X+ \\
\text { C: } Z+\end{array}$ & $\begin{array}{l}\text { B: } \mathbf{X}- \\
\text { D: } Z-\end{array}$ & $\mathrm{X}-, \mathrm{Z}-$ \\
\hline \multicolumn{3}{|c|}{ Experiment 2} \\
\hline Phase 1 & Phase 2 & Test in A, B, C, and D \\
\hline $\begin{array}{l}A: X+, Y- \\
\text { C: } Z+, W-\end{array}$ & $\begin{array}{l}\text { B: } \mathbf{X}-, \mathrm{Y}+ \\
\mathrm{D}: \mathrm{Z}-, \mathrm{W}+\end{array}$ & $\mathrm{X}-, \mathrm{Y}-, \mathrm{Z}-, \mathrm{W}-$ \\
\hline
\end{tabular}

Note-A, B, C, and D refer to the contexts in which events occurred; $X, Y, Z$, and $W$ refer to the 5 -sec conditioned stimuli; + and - designate reinforced and nonreinforced trials. 
above the magazine, behind a $10 \times 8 \mathrm{~cm}$ rectangular opening in the chamber wall. Located $0.2 \mathrm{~cm}$ behind the response key was a Magnovox (Model CK3923) color television. A computer was programmed to generate and display visual stimuli on the television screen.

The other three walls and the ceiling of each chamber were constructed of clear Plexiglas. The chambers were placed in soundand light-attenuating shells with ventilating fans that provided background noise of $62 \mathrm{~dB}(\mathrm{~A})$. A 6-W bulb was located on the wall of the shells, above the chamber ceiling. This houselight was continuously illuminated except during operation of the food hopper, during which a 6-W bulb illuminated the inside of the hopper and Purina Pigeon Grain was made available.

The contexts of the present study were provided by displaying one of four $2.5 \mathrm{~cm}$ high $\times 9.2 \mathrm{~cm}$ wide colored rectangles on the television screen. Their colors were red, green, blue, and gray. A dark circle, $1.8 \mathrm{~cm}$ in diameter, was centered in the rectangle. The stimuli were composed of small white forms, and were presented for $5 \mathrm{sec}$ in the center of the otherwise dark circle. The forms consisted of a $1.4 \times 0.2 \mathrm{~cm}$ horizontal line, and a triangle with $1.0-\mathrm{cm}$ sides and a $0.8-\mathrm{cm}$ base.

Procedure. Because of the birds' prior experience, no magazine training was necessary. The first phase consisted of 8 two-session cycles during which the birds received simple Pavlovian autoshaping training with the triangle, $\mathrm{X}$, and the horizontal line, $\mathrm{Z}$, in Contexts $A$ and $C$, respectively. In each session, the birds were initially placed in the darkened chambers, which were then illuminated by the houselight. Five seconds later, one of four colored contexts was displayed on the screen, where it remained for the rest of the session. Each session contained 18 trials in which one CS (X or Z) was presented in the context and was followed by $5 \mathrm{sec}$ of access to food. Sessions occurred in the order AC CA CA AC CA $A C A C C A$. The colors constituting the four contexts (A-D) used in this and the following phase were completely counterbalanced.

During the next session, $X$ was extinguished in Context $B$. There were 36 nonreinforced presentations of $X$ with a mean intertrial interval (ITI) of $1 \mathrm{~min}$. In the next session, $\mathrm{Z}$ was extinguished in the same manner in Context $D$.

In the following sessions, responding to $\mathrm{X}$ and $\mathrm{Z}$ was assessed in each of the four contexts. The test sessions were divided into individual cycles. Each cycle consisted of a 3-min presentation of each of the four contexts. Within each context, $\mathrm{X}$ and $\mathrm{Z}$ were presented once with a mean ITI of $1 \mathrm{~min}$. The screen was darkened for $5 \mathrm{sec}$ between presentations of each context. In order to counterbalance presentation of the contexts between subjects, for all subjects the contexts were first presented in the order red, green, blue, and gray. In the second cycle, this order was reversed. For half the subjects, $X$ was presented prior to $Z$ in each cycle; for the other half, $Z$ preceded $X$.

The first session contained two cycles. About $30 \mathrm{~min}$ after the completion of this test, the birds received another test session, similar to the first, which consisted of 4 additional cycles.

The response rate during the extinction phase prior to the test was surprisingly low. In an effort to avoid similar low levels of responding throughout the test sessions, grain was made available during a 3-min interval between each cycle for two 5-sec periods. These grain presentations occurred in the absence of any of the four explicit contextual stimuli.

\section{Results and Discussion}

Figure 1 shows the results of acquisition and extinction. Because there were no reliable differences as a function of context or CS identity, the data have been combined across the individual stimuli. It is clear that responding to the keylight CS increased gradually over the course of acquisition but then declined rapidly during

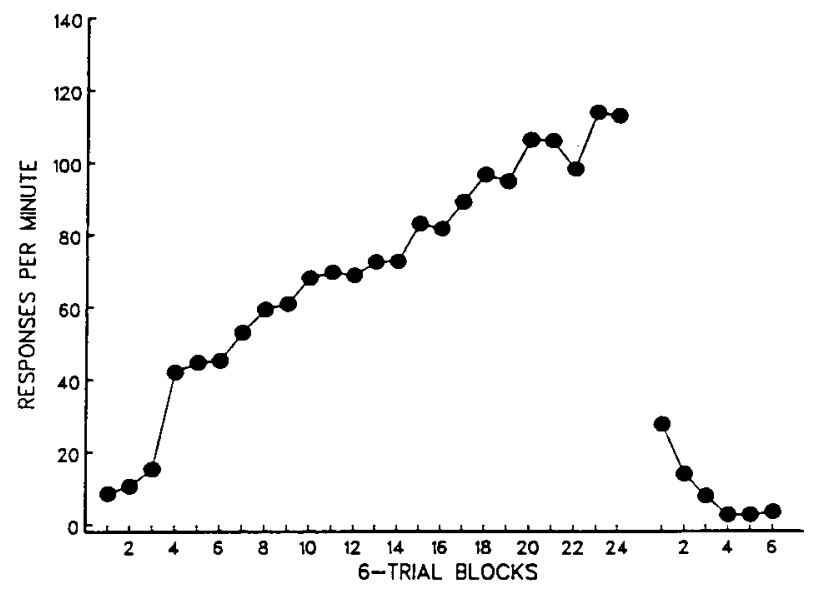

Figure 1. Responding during conditioning in Context $\mathrm{A}$ or $\mathrm{C}$, and during extinction in Context $B$ or D, in Experiment 1. Results are shown collapsed across stimulus identity.

extinction. The substantial initial disruption of responding at the beginning of extinction in a new context documents the presence of some contextual control simply as a result of acquisition. Although such disruption is not universally found in all preparations (see, e.g., Bouton \& King, 1983; Bouton \& Peck, 1989; Kaye \& Mackintosh, 1990), it agrees with the findings from several autoshaping experiments (e.g., Hall \& Honey, 1989; Honey, Willis, \& Hall, 1990).

Since the stimuli playing the role of contexts in this study were relatively localized visual cues presented on one wall of the operant chamber, it was anticipated that any excitation conditioned to them might be seen as keypecking. Nevertheless, responding during the ITI was low throughout both experimental phases. The maximum ITI rate of 1.3 responses per minute occurred on the second cycle of Phase 1. On all other cycles and during the test, ITI responding never exceeded 1.0 response per minute.

The results of testing the stimuli in the contexts are shown in Figure 2. Responding is shown collapsed across the two test sessions. Counterbalancing the order of presentation of the contexts and stimuli within the test limited the number of subjects that received each order. Thus, an investigation of the effects of order was not feasible. The results are shown separately for presentation of the original and transfer stimuli in the conditioning and the extinction contexts. Notice that a feature of the counterbalancing is that each CS served as both an original stimulus and a transfer stimulus, but for different pairs of contexts.

It is clear that responding to the original stimulus reflected its training in the contexts; the CS evoked substantially higher response rates in its conditioning context compared with its extinction context. Moreover, a similar pattern of results was observed when testing was conducted with a comparably treated transfer CS; that CS also evoked more responding in the conditioning context than in the extinction context. 


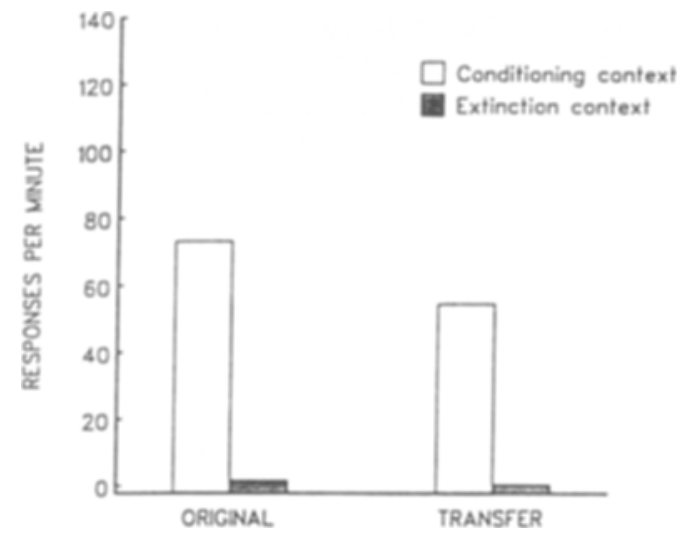

Figure 2. Responding in the conditioning and extinction contexts during the test in Experiment 1. Responding to the stimulus that was originally trained in the contexts is shown on the left. Responding to the stimulus that was similarly trained in the alternate pair of contexts is shown on the right.

These observations were confirmed in a $2 \times 2$ withinsubjects analysis of variance (ANOVA) examining context treatment (conditioning vs. extinction) and CS identity (original vs. transfer). Both main effects and their interaction were reliable $[F \mathrm{~s}(1,15)>17.9]$. Further individual comparisons were based on the methods described by Howell (1987). Those contrasts showed reliably greater responding in the conditioning context than in the extinction context for both the original and the transfer CS $[F s(1,15)>20.1]$. However, the significant interaction suggests that the difference was greater for the original training CS.

The observation of differential responding to the original CS in the conditioning and extinction contexts is consistent with the results from various other preparations (see, e.g., Bouton \& Bolles, 1979; Bouton \& Peck, 1989; Grahame et al., 1990). Even though responding was virtually eliminated during extinction in Context $B$, returning the stimulus to Context $A$ restored a substantial portion of responding. That is, responding to a stimulus that has been extinguished outside of its conditioning context can be "renewed" by returning it to the conditioning context. This demonstrates that responding to a trained and extinguished stimulus is sensitive to some aspect of the contexts in which it was presented.

The observation of differential responding to the transfer CS in the two contexts demonstrates that the power of the contexts to control performance is not confined to the CSs trained in their presence. Rather, CSs that have been similarly treated elsewhere are also controlled by the differentially treated contexts. This finding is consistent with those of Swartzentruber and Bouton (1988), and Wilson and Pearce $(1989,1990)$, who found that a context that controlled responding to one CS also controlled responding to a similarly trained transfer CS. Such transfer might indicate that a portion of the control by the contexts is attributable to their individual excitatory or in- hibitory associative strengths. Excitatory contexts might augment responding, and inhibitory contexts might reduce responding through summation with the excitation controlled by the CS. Alternatively, as a result of these training procedures, the contexts might have the ability to modulate responding to excitatory CSs in a manner that is independent of their own associative strengths.

Responding during the first test session alone revealed a somewhat different pattern of results. Although responding was still greater in the conditioning contexts than in the extinction contexts, the size of this difference was not affected by the identity of the CSs. Response rates to the original training $\mathrm{CS}$ in the conditioning and extinction contexts were 108.6 and 4.1 responses per minute, respectively, whereas response rates to the transfer CS in these contexts were 105.0 and 0.9 , respectively. A context treatment $\times$ CS identity ANOVA revealed only a main effect of context treatment $[F(1,15)=23.9]$. Neither the main effect of CS identity nor the context treatment $\times$ CS identity interaction was reliable $(F s<1)$.

The absence of a difference between the original and transfer CSs in the conditioning context during the first test session may have resulted from the presence of a response "ceiling" at the beginning of the session. The ceiling may have obscured existing differences in control over these stimuli. On the other hand, a difference between these stimuli that emerges over repeated nonreinforced trials might also reflect learning that originates during the test session. Whatever the reason for the observed difference, however, it indicates that subjects learned about specific context/CS combinations during training. This difference might indicate operation of a configural process in which the particular CS/context compounds serve as the functional stimuli. Alternatively, it could suggest that the context comes to set the occasion for specific CS-US associations.

\section{EXPERIMENT 2}

The extent to which the contexts influenced responding to the original and transfer CSs suggested that both CS-independent and CS-specific learning processes contribute to control of an extinguished CS. In Experiment 2, the same strategy was used to compare the mechanisms of control over CSs that have different conditioning histories (see Table 1). One CS (X) was first reinforced in Context $\mathrm{A}$ and then nonreinforced in Context B. Concurrently with this training, another $\mathrm{CS}$ (Y) was nonreinforced in Context A prior to being reinforced in Context $B$. This procedure thus consists of an initial phase of $\mathrm{X}+/ \mathrm{Y}$ - discrimination training in Context $\mathrm{A}$, followed by a phase in which the reinforcement contingencies are reversed, $\mathrm{X}-/ \mathrm{Y}+$, in Context $\mathrm{B}$. At the same time, two additional CSs, $Z$ and $W$, were treated in the same manner as were $\mathrm{X}$ and $\mathrm{Y}$, but in Contexts $\mathrm{C}$ and $\mathrm{D}$.

Contextual control over each CS was examined by testing the CSs in their respective training contexts. Evidence of control would be suggested by responding in a manner that was appropriate to the training received in each 
context. The extent to which any observed contextual control was specific to the CSs that were originally trained in them was assessed by examining the contexts' ability to affect responding to the stimuli that had been treated similarly in the alternate pair of contexts. If, for example, Contexts $\mathrm{A}$ and $\mathrm{B}$ control responding to $\mathrm{X}$ and $\mathrm{Y}$ through a mechanism that is independent of the CS identity, they should also influence responding to $\mathrm{Z}$ and $\mathrm{W}$. On the other hand, if control is CS specific, A and B should have little influence on responding to $Z$ and $W$. Finally, because $\mathrm{Z}$ and $\mathrm{W}$ received different training histories, any difference in the extent to which responding to $\mathrm{Z}$ and $\mathrm{W}$ is affected by $\mathrm{A}$ and $\mathrm{B}$ would indicate that the degree to which CS-independent and CS-specific processes contribute to control depends on the training history of the CS.

\section{Method}

Subjects and Apparatus. The subjects were 16 female Carneaux pigeons about 1 year old. They had participated in another autoshaping experiment with stimuli different from those used here. The housing, maintenance conditions, and apparatus were the same as in Experiment 1. Two CSs were used in addition to the triangle and horizontal line stimuli. These consisted of a $0.8 \times 0.8 \mathrm{~cm}$ white square containing a $4 \times 4$ matrix of $1-\mathrm{mm}$ black dots, and a 1.4 $\times 0.2 \mathrm{~cm}$ vertical white line.

Procedure. Because of the birds' prior experience, no magazine training was necessary. The first phase consisted of five two-session cycles during which subjects received reinforced presentations of $\mathrm{X}$ and nonreinforced presentations of $\mathrm{Y}$ in Context $\mathrm{A}$, and reinforced and nonreinforced presentations of $\mathrm{Z}$ and $\mathrm{W}$ in Context $\mathrm{C}$. Within each session, there were 18 reinforced trials and 18 nonreinforced trials. The trials were randomly presented with a mean ITI of $1 \mathrm{~min}$. One session from each cycle occurred in each context, in the order AC CA CA AC CA. For all subjects, $X$ and $Y$ consisted of the triangle and vertical line, respectively; $Z$ and $W$ were the square and horizontal line, respectively. The four colors constituting the contexts in Phases 1 and 2 were completely counterbalanced.

Phase 2 consisted of two two-session cycles, during which the reinforcement contingencies trained in Phase 1 were reversed in Contexts B and D. Each session contained 36 nonreinforced presentations of $\mathrm{X}$ or $\mathrm{Z}$, and 36 reinforced presentations of $\mathrm{Y}$ or $\mathrm{W}$. The sessions were conducted in the order BD DB.

In the following session, responding to all four stimuli in each of the four contexts was assessed following the procedures outlined in Experiment 1. The test session consisted of two cycles. Within each cycle, each context was presented for $5 \mathrm{~min}$, during which each stimulus was presented once with a mean ITI of $1 \mathrm{~min}$. The screen was dark for $5 \mathrm{sec}$ between contexts. As in Experiment 1, two 5 -sec grain presentations occurred prior to each cycle during a 3-min period while the screen remained dark.

\section{Results and Discussion}

The left side of Figure 3 shows responding to the reinforced and nonreinforced stimuli over the course of Phase 1 discrimination training in Contexts $\mathrm{A}$ and $\mathrm{C}$. Because there were no differences as a function of the identity of the stimuli or contexts, the data are shown collapsed across these factors. The right side of the figure shows responding during Phase 2 , when the reinforcement contingencies were reversed in Contexts B and D. As in Experiment 1 , presenting the stimuli in novel contexts pro-

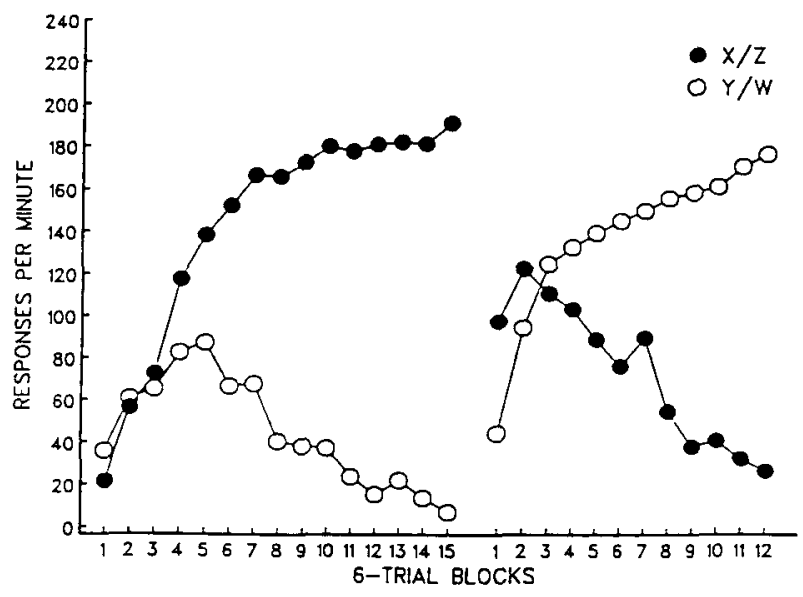

Figure 3. Responding during Phases 1 and 2 of Experiment 2. Stimuli $X$ and $Z$ were reinforced in Phase 1 and nonreinforced during Phase 2; $Y$ and $W$ were nonreinforced in Phase 1 and reinforced in Phase 2. For each stimulus, Phases 1 and 2 occurred in different contexts.

duced substantial disruption of the original training at the outset of this phase. Disrupted performance following a change in context is also observed during discrimination training with operant procedures (see, e.g., Thomas, McKelvie, \& Mah, 1985), and provides one indication that responding to the stimuli was sensitive to the context in which the initial phase was conducted.

Responding during the ITI was low throughout both phases and the test. The highest rate, 7.2 responses per minute, seen during the initial cycle of Phase 1 , was due mainly to 1 subject. Responding decreased over the remaining cycles. The mean rate of ITI responding during the test session was 0.7 responses per minute.

The data from the test session, in which the original and transfer stimuli were presented in the Phase 1 and Phase 2 contexts, are shown in Figure 4. As in Experiment 1 , because of the limited number of subjects, counterbalancing the presentation order of the contexts and stimuli made an analysis of these potential effects inappropriate. The results are shown collapsed across the identity of contexts and stimuli. Note that each CS served as an original and transfer stimulus, but in different pairs of contexts.

The left panel shows the influence of the contexts over responding to the CSs that were originally trained in them. Within this panel, the data are further separated with respect to the treatment of the CSs. The left pair of bars shows responding to the CSs that were reinforced in Phase 1 and nonreinforced in Phase $2(\mathrm{X} / \mathrm{Z})$; the right pair of bars shows responding to the CSs that were nonreinforced in Phase 1 and reinforced in Phase 2 (Y/W). It is clear that the contexts controlled responding in a manner that was appropriate to the treatments originally received in them. For each stimulus, responding in the original context of reinforcement was higher than that seen in the context of nonreinforcement. 


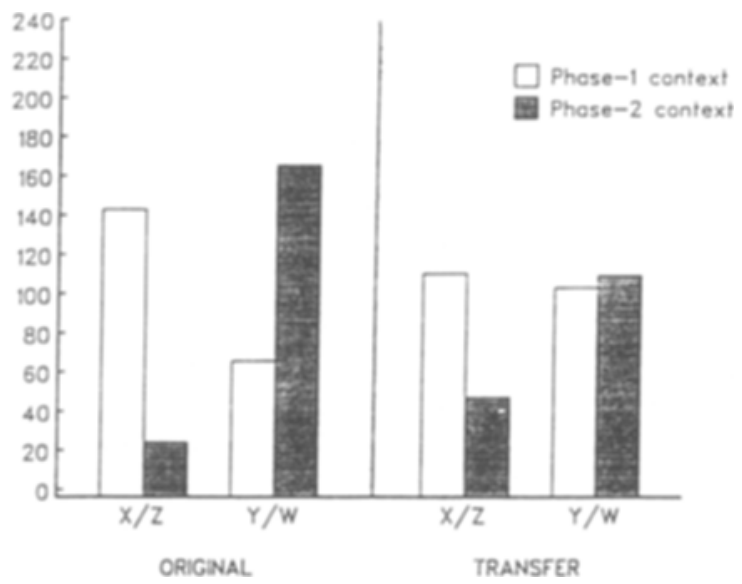

Figure 4. Responding during the test in Experiment 2. The left panel shows responding to the original context/CS combinations that were experienced during training. The right panel shows responding to the CSs that were trained in the alternate pair of contexts. Note that each stimulus provided original and transfer CS data. Results are shown collapsed across the identity of the similarly trained stimuli; $X$ and $Z$ were reinforced in Phase 1 and nonreinforced in Phase 2; $Y$ and $W$ were nonreinforced in Phase 1 and reinforced in Phase 2.

The extent to which the contexts controlled responding to the transfer stimuli is shown in the right panel, where the results are also separated with respect to the training that the transfer stimuli had received. Responding to the transfer stimuli that were conditioned in Phase 1 and extinguished in Phase $2(X / Z)$ was reliably greater in the Phase 1 context than in the Phase 2 context. As in Experiment 1 , this suggests that the contexts acquired an ability to control performance in response to CSs without regard to the specific identity of the CSs. However, responding to the transfer stimuli that were nonreinforced prior to conditioning $(\mathrm{Y} / \mathrm{W})$ was not differentially influenced by the two contexts. This suggests that the extent to which CS-independent and CS-specific processes contribute to control may depend on the training history of the CS.

These observations were confirmed with a withinsubjects ANOVA examining context treatment (Phase 1 vs. Phase 2), CS identity (original vs. transfer), and CS treatment $(\mathrm{X} / \mathrm{Z}$ vs. $\mathrm{Y} / \mathrm{W})$. The analysis revealed a significant main effect of context treatment $[F(1,15)=6.33]$, as well as significant context treatment $\times C S$ identity and context treatment $\times$ CS identity $\times$ CS treatment interactions $[F \mathrm{~s}(1,15)>26.8]$. Simple context treatment $X$ CS treatment interactions assessed the extent to which the contextual control depended on the training history of the CSs. These interactions were significant for both the original CSs $[F(1,23)=55.3]$ and the transfer CSs $[F(1,23)=$ 5.6], which suggests that the manner in which the contexts influenced responding to the stimuli depended on the order in which reinforcement and nonreinforcement had occurred. However, the reliable context treatment $x$ CS identity $\times$ CS treatment interaction suggests that the extent to which the contexts influenced responding depended on whether original or transfer context/CS compounds were being tested.

Further contrasts compared the extent of contextual control over each of the original and transfer CSs. The levels of responding in the two contexts were reliably different for both of the original CSs $\left[F_{\mathrm{s}}(1,47)>28.1\right]$, which confirms that responding to each CS in its original context reflected the treatment received there. The extent to which the contexts influenced responding to the transfer CSs, however, revealed a different pattern. Although responding to the trained and extinguished transfer stimulus was reliably greater in the Phase 1 than in the Phase 2 context $[F(1,47)=11.3]$, responding to the transfer stimulus that had been nonreinforced prior to conditioning was not differentially affected $[F(1,47)<1]$. This suggests that the extent of transfer depended on the training history of the transfer stimulus.

To summarize, contextual control of responding to the original CSs suggests that responding was sensitive to some feature of the contexts in which they were presented. Furthermore, a comparison of the pattern of responding to that for the original and transfer stimuli suggests that both CS-independent and CS-specific processes contribute to this contextual control. The contribution of a CSspecific process is suggested by the observation that control of responding to the original stimuli was stronger than control of responding to the transfer stimuli. Because the original and transfer stimuli were treated similarly, weaker control of the transfer stimuli reveals the importance of the original context/CS combinations. On the other hand, the observation of transfer to the CS that had undergone training and extinction suggests that contextual control also involves a process that is independent of the CS identity. However, the finding that transfer was not observed to the CS that had been nonreinforced prior to conditioning suggests that any CS-independent property acquired by the contexts operates differently on stimuli that have received different training histories.

\section{GENERAL DISCUSSION}

In these experiments, the nature of contextual control was examined in two paradigms in which separate phases of CS reinforcement and nonreinforcement occurred in different contexts. In Experiment 1, control of responding to a CS was examined when the CS was conditioned in one context (A) and then extinguished in a different context (B). When the CS was then presented in A and $B$ following extinction, responding was still strong in $\mathbf{A}$, and nearly absent in $\mathbf{B}$, thus demonstrating sensitivity to some feature of the training contexts. However, another CS that had been conditioned and extinguished in a different pair of contexts was also influenced by $A$ and $B$ in a manner similar to that for the original CS, but to a lesser degree, suggesting that contextual control was at least partly mediated by a process that did not depend on the specific identity of the CS. 
In Experiment 2, the same strategy was employed to examine the nature of contextual control of two CSs that had received different reinforcement treatments. One CS was first reinforced in $\mathrm{A}$ and then nonreinforced in $\mathrm{B}$, and another $\mathrm{CS}$ was first nonreinforced in $\mathrm{A}$ and then reinforced in B. When the CSs were then tested in A and B, responding to each $C S$ reflected the treatments that were given in each context, suggesting that responding to both CSs was sensitive to the training contexts. However, when contextual control by A and B was assessed on transfer CSs that had received reinforcement treatments similar to those for the original CSs, the contexts transferred control only to the CS that was reinforced and then nonreinforced. The contexts did not influence responding to the CS that was nonreinforced prior to reinforcement.

The overall pattern of results suggests that several processes contribute to the contextual control of responding to CSs. For example, regardless of the training history of the CSs, the contexts exerted stronger control of responding to the original CSs than of responding to the similarly trained transfer CSs. Incomplete transfer suggests that control is at least partly mediated by a CSspecific process. Several mechanisms have been suggested that would account for this result. In configural or unique cue mechanisms, for example, control arises because each context/CS combination is perceived as a unique compound during training and testing. Weaker discrimination performance when the contexts and CSs are presented in novel combinations during the test reflects the importance of the original compounds' unique properties. An alternative CS-specific process is perhaps best characterized by a hierarchical "occasion setting" mechanism (e.g., Holland, 1983, 1985), in which the contexts control responding to specific CS-US relations. Unlike the configural view, this conceptualization places the context in a different role from that of the CS. However, the present results do not distinguish between the contributions of these alternative mechanisms.

Although control of responding to the original CSs was strongest, in both experiments control of responding to the transfer stimulus that had undergone conditioning and extinction was also observed. This suggests that the contexts had acquired an ability to control responding through a mechanism that is not dependent on the specific CS identity. This differential control by the Phase 1 and Phase 2 contexts would have arisen from the different treatments that were administered in each phase. For example, in Experiment 2, although CS reinforcement and nonreinforcement occurred in both contexts, the number of reinforced and nonreinforced trials differed in each phase. Perhaps more importantly, CS reinforcement in the Phase 1 context comprised initial acquisition, whereas the CS reinforcement treatment in Phase 2 followed an initial phase in which the CS had been nonreinforced. Likewise, the nonreinforcement treatments differed in whether they consisted of initial nonreinforced CS preexposure or extinction of a conditioned response. Finally, the two contexts differed simply in the temporal sequence in which they were experienced. Although it is unclear exactly how these differences might affect the acquisition of different CS-independent contextual properties, it is conceivable that these contexts would have differential influences on responding to transfer CSs.

Several mechanisms might account for the ability of a context to influence responding in a transfer test. In one, it is assumed that contexts and CSs both acquire associations with the US. Performance is then determined by summation of these associations during the test. However, it is interesting to note that the present contexts evoked little keypecking on their own, which might indicate that the contexts were only weakly excitatory or that they evoked a different conditioned response. Little is currently known about how stimuli that evoke different responses would summate in performance. In any event, although associative summation may provide a sufficient account for the transfer observed to the trained and extinguished CS in both experiments, a similar degree of summation would also be expected to the nonreinforced and then reinforced CS in Experiment 2. A simple associative account for the results of this experiment thus appears incomplete.

Alternatively, the contexts may have acquired the ability to modulate responding to the transfer CS independently of the contexts' simple US associations. The observation in Experiment 2 that transfer depended on the training history of the transfer CSs suggests a parallel with the results from other modulatory research. Investigators using several different Pavlovian preparations have found that the extent of transfer depends almost invariably on the nature of the training history of the transfer target stimulus (e.g., Bouton \& King, 1986; Holland, 1989, 1991; Rescorla, 1985; Wilson \& Pearce, 1990). Unfortunately, because the conditions under which a stimulus will acquire such a modulatory ability are unclear, it is difficult to determine how the individual conditioning treatments administered in each context may have influenced the contextual modulatory strength. However, the degree to which transfer depended on the nature of the transfer CS's training in the present preparation might suggest the involvement of a modulatory mechanism similar to that seen in other preparations.

Although transfer of control appears to require the contribution of some CS-independent process, transfer could also result from generalization between the original and transfer stimuli. The difficulty in assessing the contribution of stimulus generalization to transfer has been an issue in several paradigms (see, e.g., Davidson \& Rescorla, 1986; Wilson \& Pearce, 1989). In the present experiments, some degree of generalization seems likely. One popular account of the role that generalization might play in conditioning has recently been proposed by Pearce (1987). According to his theory, conditioning occurs solely to configured compounds of all stimulus elements present on a given trial. The extent to which a different compound subsequently evokes a response depends on the similarity of that compound to the compound originally involved in training. In the present experiments, for ex- 
ample, conditioning of $\mathrm{CS} \mathrm{X}$ in Context $\mathrm{A}$ would result in an excitatory $A X$ compound. Subsequent responding to $\mathrm{X}$ in Context B would reflect the similarity of BX and $A X$, and as a result of nonreinforced $B X$ presentations during extinction, BX would acquire inhibitory strength of sufficient magnitude to offset the generalized excitation from AX. Responding to the CX and DX compounds during the subsequent transfer tests would depend on the similarities and associative strengths of all pretrained compounds containing the $\mathrm{C}, \mathrm{D}$, and $\mathrm{X}$ elements.

Following the rules specified in Pearce's model, a simulation of the present experiments does, in fact, predict substantial transfer to the unique transfer compounds on the test. Consistent with the empirical observations, the trained and extinguished CSs are predicted to show greater responding in the Phase 1 contexts than in the Phase 2 contexts. On the other hand, a similar pattern of responding to the transfer compounds containing the CSs that were first nonreinforced prior to reinforcement is predicted. Differential responding to these compounds was not found.

One path that some generalization models have taken is to consider the associative strength of the elements of a compound when determining the degree to which responding will generalize. Blough (1975), for example, proposed a model of generalization in which inhibitory generalization gradients are steeper than those for excitation. To the extent that extinction involves acquisition of an inhibitory component, such an assumption could predict different patterns of responding to transfer compounds containing elements with different conditioning histories. In the present Experiment 2, this would amount to a greater generalization decrement when the extinguished CSs $(X / Z)$ are tested outside of the original extinction context, and a relatively small decrement for the recently conditioned CSs $(\mathrm{Y} / \mathrm{W})$ when they are tested outside of their conditioning contexts. However, note that even with this assumption, stimuli with different histories would still show a similar pattern of responding when tested in the two transfer compounds. For example, although $\mathrm{Z}$ and $\mathrm{W}$ might show different amounts of decrement upon removal from their original training contexts (C and D), the pattern of differential responding in Contexts A and B should be similar. Although a generalization model emphasizing the effect of the conditioning history might provide a more accurate account of some features of the present results, it is not easy to see how this assumption would predict the specific pattern of responding found here.

The pattern of responding to the original context/CS combinations during the test in Experiment 2 is consistent with the results of a number of experiments by Thomas and his colleagues (e.g., Thomas, Curran, \& Russell, 1988; Thomas et al., 1985; Thomas, McKelvie, Ranney, \& Moye, 1981), who have examined contextual conditional control of discriminative responding in an operant preparation. As in the Pavlovian preparation used here, conditional control has often been observed with a procedure that involves a single reversal of the reinforcement contingencies. Unfortunately, there are currently no transfer data from the operant preparation that would help determine the extent to which such conditional control is stimulus specific.

However, it is interesting to note that if a mechanism that is not stimulus specific contributes to single reversal learning, this would imply that different processes are involved in single reversal and intermixed conditional discrimination learning. During single-reversal training, the first- and second-phase contexts acquire differential properties that are independent of the specific stimulus identities. Responding during the test is partly controlled by these different properties. On the other hand, during multiple discrimination reversals, or intermixed training, the contexts receive operationally equivalent treatments, and solution of the discrimination requires learning about the specific context/CS combinations. Learning must therefore involve a stimulus-specific hierarchical or configural mechanism. Although explicit comparisons across paradigms should perhaps be viewed with some caution, the existing parallels suggest that common processes may be involved in operant and Pavlovian conditional control.

In summary, these experiments provide evidence that several different processes contribute to the contextual control observed following a single reversal of the reinforcement contingencies. Although control of responding to the original CSs involves a degree of CS specificity, reliable transfer to a CS that was trained in a different set of contexts suggests that the contexts may acquire differential modulatory properties during training. Furthermore, the observation that the extent of transfer depended on the training history of the CS suggests that this modulatory process may be similar to that involved in other Pavlovian modulatory paradigms.

\section{REFERENCES}

BLough, D. S. (1975). Steady state data and a quantitative model of operant generalization and discrimination. Joumal of Experimental Psychology: Animal Behavior Processes, 104, 3-21.

Bouton, M. E. (1991). Context and retrieval in extinction and in other examples of interference in simple associative learning. In $L$. W. Dachowski \& C. F. Flaherty (Eds.), Current topics in animal learning: Brain, emotion, and cognition (pp. 25-53). Hillsdale, NJ: Erlbaum.

Bouton, M. E., Bolles, R. C. (1979). Contextual control of the extinction of conditioned fear. Learning \& Motivation, 10, 445-466.

Bouton, M. E. , \& KING, D. A. (1983). Contextual control of the extinction of conditioned fear: Tests for the associative value of the context. Joumal of Experimental Psychology: Animal Behavior Processes, 9, 248-265.

Bouton, M. E., KING, D. A. (1986). Effect of context on performance to conditioned stimuli with mixed histories of reinforcement and nonreinforcement. Journal of Experimental Psychology: Animal Behavior Processes, 12, 4-15.

Bouton, M. E., \& PeCk, C. A. (1989). Context effects on conditioning, extinction, and reinstatement in an appetitive conditioning preparation. Animal Learning \& Behavior, 17, 188-198.

Bouton, M. E. , Swartzentruber, D. (1986). Analysis of the associative and occasion-setting properties of contexts participating in a Pavlovian discrimination. Journal of Experimental Psychology: Animal Behavior Processes, 12, 333-350. 
Bouton, M. E., \&wartzentruber, D. (1989). Slow reacquisition following extinction: Context, encoding, and retrieval mechanisms. Journal of Experimental Psychology: Animal Behavior Processes, 15. 43-53.

DAvidson, T. L., \& Rescorla, R. A. (1986). Transfer of facilitation in the rat. Animal Learning \& Behavior, 14, 380-386.

Grahame, N. J., Hallam, S. C., Geier, L., \& Miller, R. R. (1990). Context as an occasion setter following either CS acquisition and extinction or CS acquisition alone. Learning \& Motivation, 21, 237-265.

Grau, J. W., Rescorla, R. A. (1984). Role of context in autoshaping. Joumal of Experimental Psychology: Animal Behavior Processes, 10, 324-332.

HALl, G., \& HoNEY, R. C. (1989). Contextual effects in conditioning, latent inhibition, and habituation: Associative and retrieval functions of contextual cues. Joumal of Experimental Psychology: Animal Behavior Processes, 15, 232-241.

Hall, G., \& Honey, R. C. (1990). Context-specific conditioning in the conditioned emotional response procedure. Joumal of Experimental Psychology: Animal Behavior Processes, 16, 271-278.

Holland, P. C. (1983). Occasion setting in Pavlovian feature positive discriminations. In M. L. Commons, R. J. Herrnstein, \& A. R. Wagner (Eds.), Quantitative analyses of behavior: Discrimination processes (Vol. 4, pp. 183-206). New York: Ballinger.

Holland, P. C. (1985). The nature of conditioned inhibition in serial and simultaneous feature negative discriminations. In R. R. Miller \& N. E. Spear (Eds.), Information processing in animals: Conditioned inhibition (pp. 267-297). Hillsdale, NJ: Erlbaum.

Holland, P. C. (1989). Transfer of negative occasion setting and conditioned inhibition across conditioned and unconditioned stimuli. Journal of Experimental Psychology: Animal Behavior Processes, 15, 311-328.

Holland, P. C. (1991). Acquisition and transfer of occasion setting in operant feature positive and feature negative discriminations. Learning \& Motivation, 22, 366-387.

Honey, R. C., Willis, A., \& Hall, G. (1990). Context specificity in pigeon autoshaping. Learning \& Motivation, 21, 125-136.

HowELL, D. C. (1987). Statistical methods for psychology. Boston: Duxbury Press.

Kaye, H., \&ackintosh, N. J. (1990). A change of context can enhance performance of an aversive but not of an appetitive conditioned response. Quarterly Journal of Experimental Psychology, 42B, 113-134.
Lovibond, P. F., Preston, G. C., \& Mackintosh, N. J. (1984). Context specificity of conditioning, extinction, and latent inhibition. Joumal of Experimental Psychology: Animal Behavior Processes, 10, 360-375.

Pearce, J. M. (1987). A model for stimulus generalization in Pavlovian conditioning. Psychological Review, 94, 61-73.

Rescorla, R. A. (1985). Conditioned inhibition and facilitation. In R. R. Miller \& N. E. Spear (Eds.), Information processing in animals: Conditioned inhibition (pp. 299-326). Hillsdale, NJ: Erlbaum.

Rescorla, R. A., Durlach, P. J., \& Grau, J. W. (1985). Contextual learning in Pavlovian conditioning. In P. D. Balsam \& A. Tomie (Eds.), Context and learning (pp. 23-56). Hillsdale, NJ: Erlbaum.

Rescorla, R. A., WAgner, A. R. (1972). A theory of Pavlovian conditioning: Variations in the effectiveness of reinforcement and nonreinforcement. In A. H. Black \& W. F. Prokasy (Eds.), Classical conditioning II: Current research and theory (pp. 64-99). New York: Appleton-Century-Crofts.

SWARTZENTRUBER, D. (1991). Blocking between occasion setters and contextual stimuli. Joumal of Experimental Psychology: Animal Behavior Processes, 17, 163-173.

Swartzentruber, D., \& Bouton, M. E. (1988). Transfer of positive contextual control across different conditioned stimuli. Bulletin of the Psychonomic Society, 26, 569-572.

Thomas, D. R., Curran, P. J., \& Russell, R. J. (1988). Factors affecting conditional discrimination learning by pigeons: II. Physical and temporal characteristics of stimuli. Animal Learning \& Behavior, 16, 468-476.

Thomas, D. R., McKelvie, A. R., \& Mah, W. L. (1985). Context as a conditional cue in operant discrimination reversal learning. Joumal of Experimental Psychology: Animal Behavior Processes, 11, 317-330.

Thomas, D. R., McKelvie, A. R., Ranney, M., \& Moye, T. B. (1981). Interference in pigeons' long-term memory viewed as a retrieval problem. Animal Learning \& Behavior, 9, 581-586.

Wilson, P. N., \& Pearce, J. M. (1989). A role for stimulus generalization in conditional discrimination learning. Quarterly Joumal of Experimental Psychology, 41B, 243-273.

Wilson, P. N., \& PEARCE, J. M. (1990). Selective transfer of responding in conditional discriminations. Quarterly Joumal of Experimental Psychology, 42B, 41-58.

(Manuscript received November 25, 1991; revision accepted for publication May 22, 1992.) 